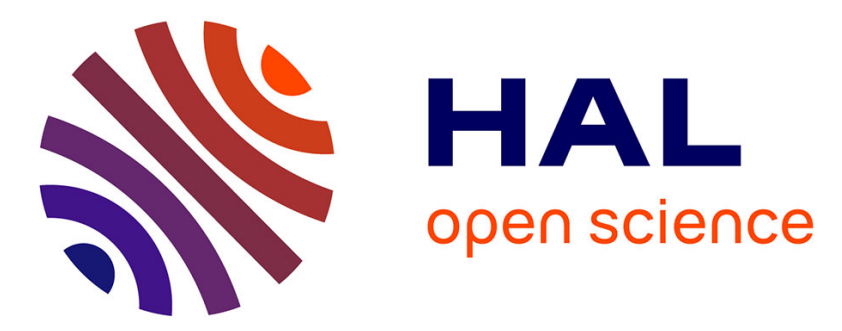

\title{
Search for interference effects in electron impact ionization of aligned hydrogen molecules
}

A Senftleben, T Pflüger, X Ren, O Al-Hagan, B Najjari, D Madison, A Dorn, J Ullrich

\section{- To cite this version:}

A Senftleben, T Pflüger, X Ren, O Al-Hagan, B Najjari, et al.. Search for interference effects in electron impact ionization of aligned hydrogen molecules. Journal of Physics B: Atomic, Molecular and Optical Physics, 2010, 43 (8), pp.81002. 10.1088/0953-4075/43/8/081002 . hal-00569896

\section{HAL Id: hal-00569896 \\ https://hal.science/hal-00569896}

Submitted on 25 Feb 2011

HAL is a multi-disciplinary open access archive for the deposit and dissemination of scientific research documents, whether they are published or not. The documents may come from teaching and research institutions in France or abroad, or from public or private research centers.
L'archive ouverte pluridisciplinaire HAL, est destinée au dépôt et à la diffusion de documents scientifiques de niveau recherche, publiés ou non, émanant des établissements d'enseignement et de recherche français ou étrangers, des laboratoires publics ou privés. 


\title{
FAST TRACK COMMUNICATION
}

\section{Search for interference effects in electron impact ionization of aligned hydrogen molecules}

\author{
A Senftleben ${ }^{1}$, T Pflüger ${ }^{1}$, X Ren ${ }^{1}$, O Al-Hagan ${ }^{2}$, B Najjari ${ }^{1}$, \\ D Madison ${ }^{2}$, A Dorn ${ }^{1}$ and J Ullrich ${ }^{1}$ \\ ${ }^{1}$ Max-Planck-Institut für Kernphysik, Saupfercheckweg 1, 69117 Heidelberg, German \\ 2 Department of Physics, Missouri University of Science \& Technology, Rolla, MO \\ USA 65409
}

\begin{abstract}
Five-fold differential cross sections (5DCS) for electron impact ionization of a diatomic molecule have been explored experimentally as a function of molecular alignment. Using $\mathrm{H}_{2}$ as a test system we exploited dissociative ionization by $200 \mathrm{eV}$ electrons to obtain the alignment of the internuclear axis. Separation of groundstate ionization from autoionization is discussed. 5DCS are investigated for the direct channel and found to be in good agreement with M3DW calculations discarding at the same time a simple two-centre interference model discussed recently in literature.
\end{abstract}

PACS numbers: $34.80 . \mathrm{Gs}$

Submitted to: J. Phys. B: At. Mol. Opt. Phys.

The complex dynamics of molecular ionization by energetic electron impact, also known as the $(e, 2 e)$ reaction, has been widely studied during the last decades. Its understanding is of paramount importance for fields such as radiation tumour therapy, the physics and chemistry of planetary atmospheres, near-stellar clouds or reactive plasmas. In general, a projectile electron knocks out a bound electron from the target leading to at least three fragments in the final state, two electrons and one ion. Especially the simple diatomic hydrogen molecule was intensely studied for a wide range of electron energies. Much research was dedicated to total cross sections and their dependence on the alignment which is given by the relative angle between the internuclear axis and the incoming electron beam (Dunn \& Kieffer 1963, Van Brunt \& Kieffer 1970). On the other hand, detailed studies on the final-state electron characteristics were performed for a wide range of kinematic settings (Weigold et al. 1973, Cherid et al. 1989, MilneBrownlie et al. 2006, Murray 2005, Staicu Cassagrande et al. 2008, Al-Hagan et al. 2008). However, the ultimate experiment exploring five-fold differential cross sections (5DCS), thus capturing the full kinematics simultaneously with controlling the molecular alignment has not been realized up to now, even though efforts have been made to do so (Takahashi et al. 2004, Takahashi et al. 2005, Bellm et al. 2010).

On the theoretical side, 5DCS have been investigated recently (Stia et al. 2003, Colgan et al. 2008, Colgan et al. 2009), finding a distinct dependence of the 
electron scattering dynamics on the alignment. Some of the observed features, especially unexpected minima in the angular spectra were attributed to interference effects, either as a consequence of the two-centre nature of $\mathrm{H}_{2}$ (Stia et al. 2003) or by coherent superposition of partial waves (Colgan et al. 2009). Traces of two-centre interference were predicted even for three-fold differential cross sections (3DCS) measured with randomly aligned molecules. Evidence for their experimental observation was reported at impact energies above $500 \mathrm{eV}$ (Staicu Cassagrande et al. 2008) and at $250 \mathrm{eV}$ (MilneBrownlie et al. 2006), but excluded in investigations below $100 \mathrm{eV}$ (Murray 2005). Thus, the subject has been discussed controversially, calling for the most stringent test of the interference hypotheses that can only be provided by alignment-dependent 5DCS.

In this work, 5DCS are presented for $200 \mathrm{eV}$ electrons colliding with hydrogen molecules which can be ionized above $15.4 \mathrm{eV}$. The general geometry of such a reaction is displayed in figure 3 (a). In most cases, the incoming projectile will lose a relatively small amount of energy $\Delta E$. Additionally, it will be deflected by a small angle $\theta_{e 1}$. The momentum vectors of the projectile before and after the collision define the scattering plane, which also contains the momentum $\vec{q}$ transferred onto the target. But the molecular alignment and the momentum of the emitted electron $\vec{p}_{e 2}$ are not bound to this plane.

In our current experiment, the long-standing shortage of experimental 5DCS has been overcome by determining the alignment of the internuclear axis from dissociation of the residual $\mathrm{H}_{2}{ }^{+}$ion in the wake of the ionizing collision. Dissociation as investigated here can take two distinct reaction pathways which are illustrated in the potential curves diagram of figure 1 . On the one hand, it is possible to populate the vibrational continuum of the $\mathrm{H}_{2}{ }^{+}$ground state. This channel is called ground-state dissociation (GSD). The second process is autoionization (AI) which proceeds in three steps: First, a doubly excited state of the neutral molecule is populated. Only the lowest-lying of these is shown in figure 1, but there is an infinite number of such levels. All of them are repulsive within the Franck-Condon region accessible from the ground state. Consequently, the excited molecule starts to dissociate (second step) where the two nuclei gain a sum kinetic energy of $A$. As long as the ionic ground state lies energetically lower, spontaneous autoionization is possible in the third step. Thereby, the emitted electron gains the energy $E_{e 2}$ equal to the difference of the two potential curves at the current internuclear separation $R$. The residual $\mathrm{H}_{2}{ }^{+}$ion can be stable if $A$ is smaller than the dissociation energy $D$. Otherwise, the ion will fragment into a proton and a neutral hydrogen atom with a kinetic energy release (KER) of $A-D$.

Deducing the molecular alignment from the emission direction of dissociation fragments implies the validity of the axial recoil approximation (Zare 1967), which is fulfilled if the $\mathrm{H}_{2}{ }^{+}$ion fragments faster than it rotates. Using the method suggested by (Wood et al. 1997) we have verified for the dissociation processes relevant here that the alignment can be determined with an uncertainty of $\pm 20^{\circ}$ or less for kinetic energy releases above $.13 \mathrm{eV}$. The set-up used to measure protons as well as the two final state electrons is an advanced reaction microscope purpose-built to study ionization 


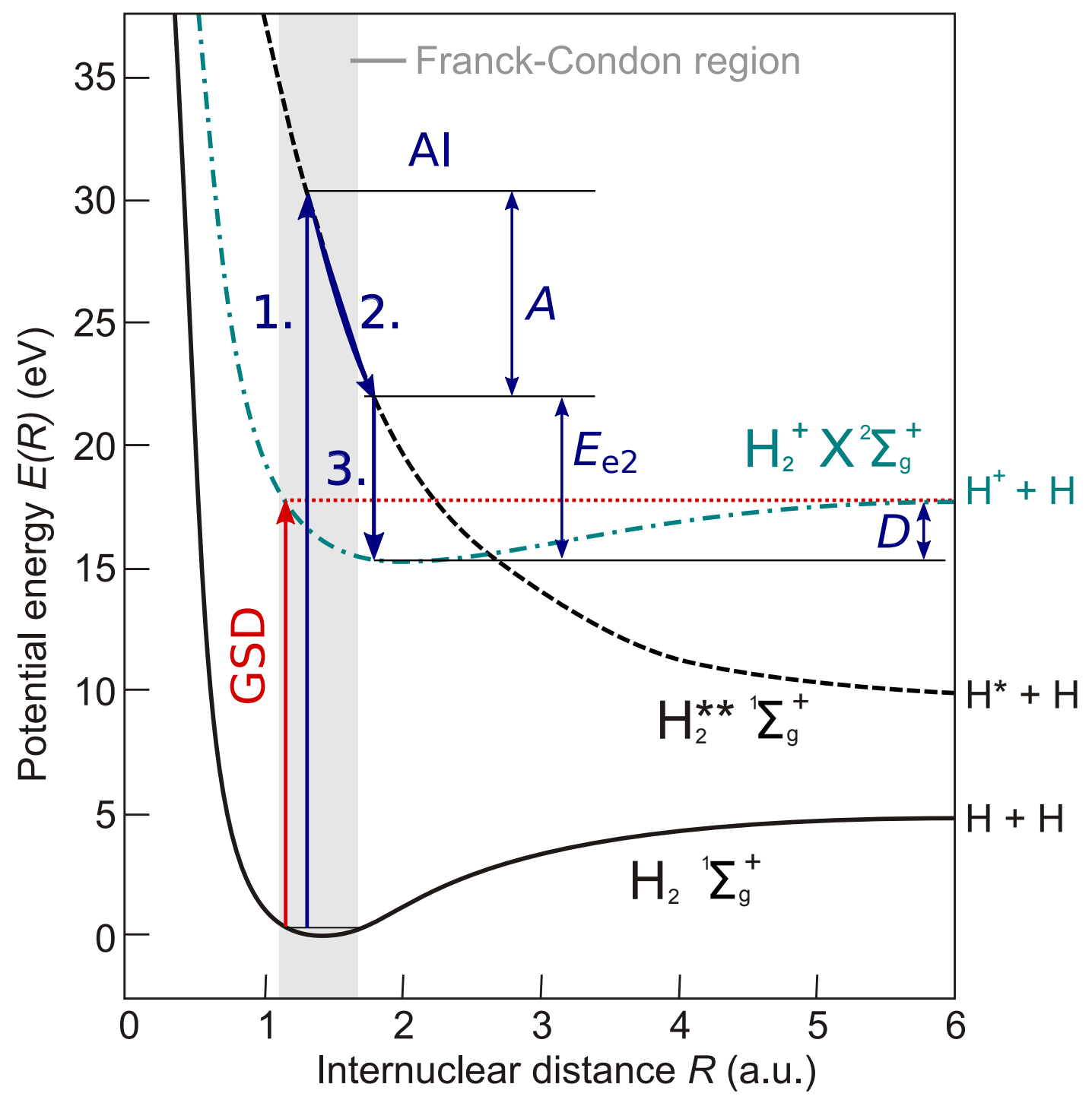

Figure 1. (colour online) Selected potential curves of $\mathrm{H}_{2}$ and $\mathrm{H}_{2}{ }^{+}$(after (Sharp 1970, Guberman 1983)) with illustration of two dissociative ionization channels: Ground-state dissociation (GSD) and autoionization (AI).

by low and medium energetic electrons as described in previous works (Dürr et al. 2007, Dorn et al. 2007). Briefly, a pulsed electron beam from a thermal source is crossed with a jet of cold hydrogen gas created by super sonic expansion. Beam and target densities are kept low enough such that ionization will occur in less than every tenth shot. Charged collision products are accelerated and guided by well-defined electric and magnetic fields towards two position and time sensitive detectors. From this, threedimensional momentum vectors of all particles can be calculated. Unlike previous studies the detector collecting ions has been significantly enlarged and additionally moved closer to the reaction point to increase the acceptance of energetic fragments stemming from dissociation. Furthermore, this structure needed to employ a central bore to allow the incoming beam to pass. This was realized with a specially designed hexagonal delay 


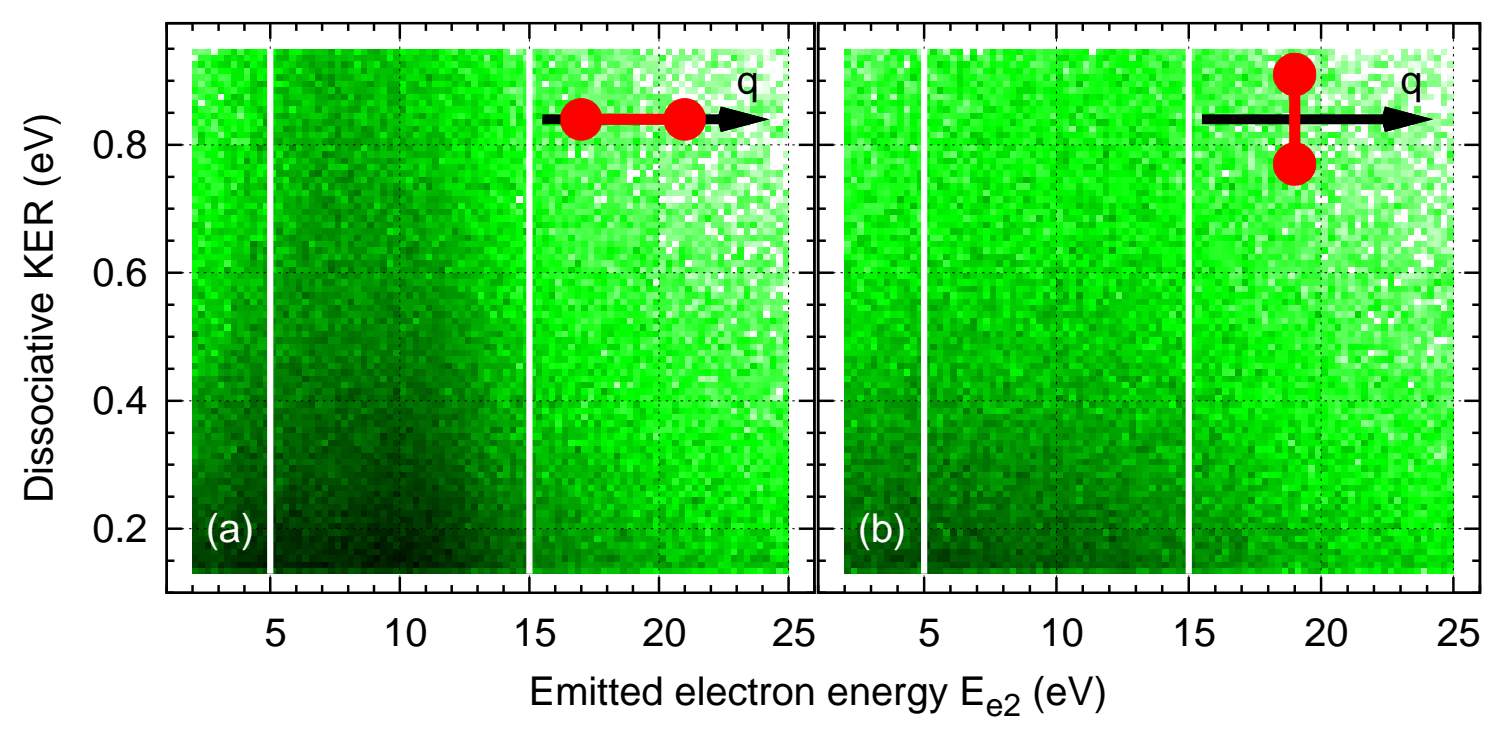

Figure 2. Measured kinetic energy released to the fragments of the dissociating $\mathrm{H}_{2}{ }^{+}$ion versus the emitted electron's energy for molecules aligned parallel (a) and perpendicular (b) to the momentum transfer $\vec{q}$. The logarithmic colour scales are identical in both images, with black representing the highest count rates. The two vertical lines indicate the energy transfer region corresponding to the excitation of autoionizing states.

line anode (Jagutzki et al. 2002) constructed around a beam tube and, thus, requiring a sophisticated method to read out the position information similar to that described by (Pedersen et al. 2009). It should be noted that neutral fragments are not detected. Since the dissociation of the $\mathrm{H}_{2}{ }^{+}$ion leads to one $\mathrm{H}$ atom, its momentum has to be derived through momentum conservation. With the electric and magnetic field settings used we have been able to detect protons emerging from dissociation of $\mathrm{H}_{2}{ }^{+}$over the complete solid angle for a kinetic energy release of up to $1 \mathrm{eV}$. The electron detector also contains a hole in its active area where the beam dump is located. As a consequence of this, electrons emitted under a small angle with respect to the beam direction cannot be detected.

The two dissociation channels can be distinguished experimentally through the KER of the heavy fragments, which is derived by doubling the measured energy of the proton, and the emitted electron's energy $E_{e 2}$, as illustrated in figure 2. This method was demonstrated in ion impact ionization of $\mathrm{H}_{2}$ (Laurent et al. 2006) where the experimental values were compared to calculated energies. In electron impact studies, channel-selective KER distributions have also been extensively studied and well understood (Van Brunt \& Kieffer 1970, Edwards et al. 1990, Van Zyl \& Stephene 1994): While GSD is the overwhelmingly dominating channel at KERs close to zero its relative contribution rapidly drops below the AI rate around $1 \mathrm{eV}$. Therefore, in the energy range studied in this work, it is not possible to separate the two processes through KER alone. To examine the channel-dependent behaviour of $E_{e 2}$ we consider 
energy conservation for our reaction. The projectile's energy loss $\Delta E$ is composed as follows:

$$
\Delta E=E_{D}+E_{e 2}+K E R
$$

where $E_{D}=18 \mathrm{eV}$ is the energy of the first dissociation limit of $\mathrm{H}_{2}{ }^{+}$above the ground state of the neutral molecule. If we neglect the kinetic energy release because it is in most cases smaller than $1 \mathrm{eV}$, we see that $E_{e 2}$ is linearly linked with the energy loss which is continuous for direct ionization but takes discrete values for excitation. Hence, events from autoionization should employ energies for the emitted electron that can be associated with the energy transfers necessary to populate doubly-excited states of $\mathrm{H}_{2}$. For example, the lowest lying of these levels (shown as ${ }^{1} \Sigma_{g}^{+}$in figure 1) is accessible in the Franck-Condon region between 24 and $34 \mathrm{eV}$ energy transfer. It has been shown earlier that this is the main contributing autoionizing state in electron impact excitation (Edwards \& Zheng 2001). In our case, this energy transfer region corresponds to a secondary electron energy between roughly 5 and $15 \mathrm{eV}$, which is approximately where we see an increased count rate in figure 2 (a), especially for KERs above $.5 \mathrm{eV}$. However, most of the counts with larger KERs are situated between $E_{e 2}=5$ and $12 \mathrm{eV}$. Changing the direction of the molecular axis to aligned perpendicular to the momentum transfer (figure 2 (b)) the count rate rapidly drops with larger kinetic energy releases at any value of $E_{e 2}$, showing that for this geometry GSD is the dominant process. From this we can already estimate that the autoionization rate depends more strongly on the molecular alignment.

In figure 3 (b) to (d) the emission direction of the protonic fragment is plotted in the scattering plane system as defined in figure 3 (a) for different electron energies $E_{e 2}$ and, consequently, energy losses $\Delta E$. Figure 3 (c) comprises the region where autoionization is predicted due to the increased large KER count rates in figure 2 (a). It clearly exhibits the strongest anisotropy of all distributions. We will postpone further discussion of autoionization to a future publication. However, for the energy ranges where GSD should be the sole contributing process, an increased rate for molecular alignment parallel to the momentum transfer has been measured as well (see figure 3 (b) and $(\mathrm{d}))$. To further investigate these findings, figure 4 displays an exemplary 5DCS spectrum for ionization into the ground state of $\mathrm{H}_{2}{ }^{+}$. Hereby, the second electron's polar angle distribution is plotted for emission into the scattering plane at an energy of $3.5 \mathrm{eV}$ while the scattering angle is fixed to $16^{\circ}$. Three distinct molecular alignments are selected. Here, the internuclear axis is aligned in the scattering plane at angles of $0^{\circ}, 45^{\circ}$ and $90^{\circ}$ relative to the momentum transfer. Other geometries will be discussed in a subsequent publication.

Ground-state ionization has the advantage that its 5DCS can be calculated by state-of-the-art theoretical models. Here we present cross-sections obtained with the molecular 3-body distorted wave (M3DW) method (Gao et al. 2006). They are displayed in figure 4 (a). The model has been used to normalize the experimental data at the theoretical maximum for an angle of $45^{\circ}$ between internuclear axis and momentum 

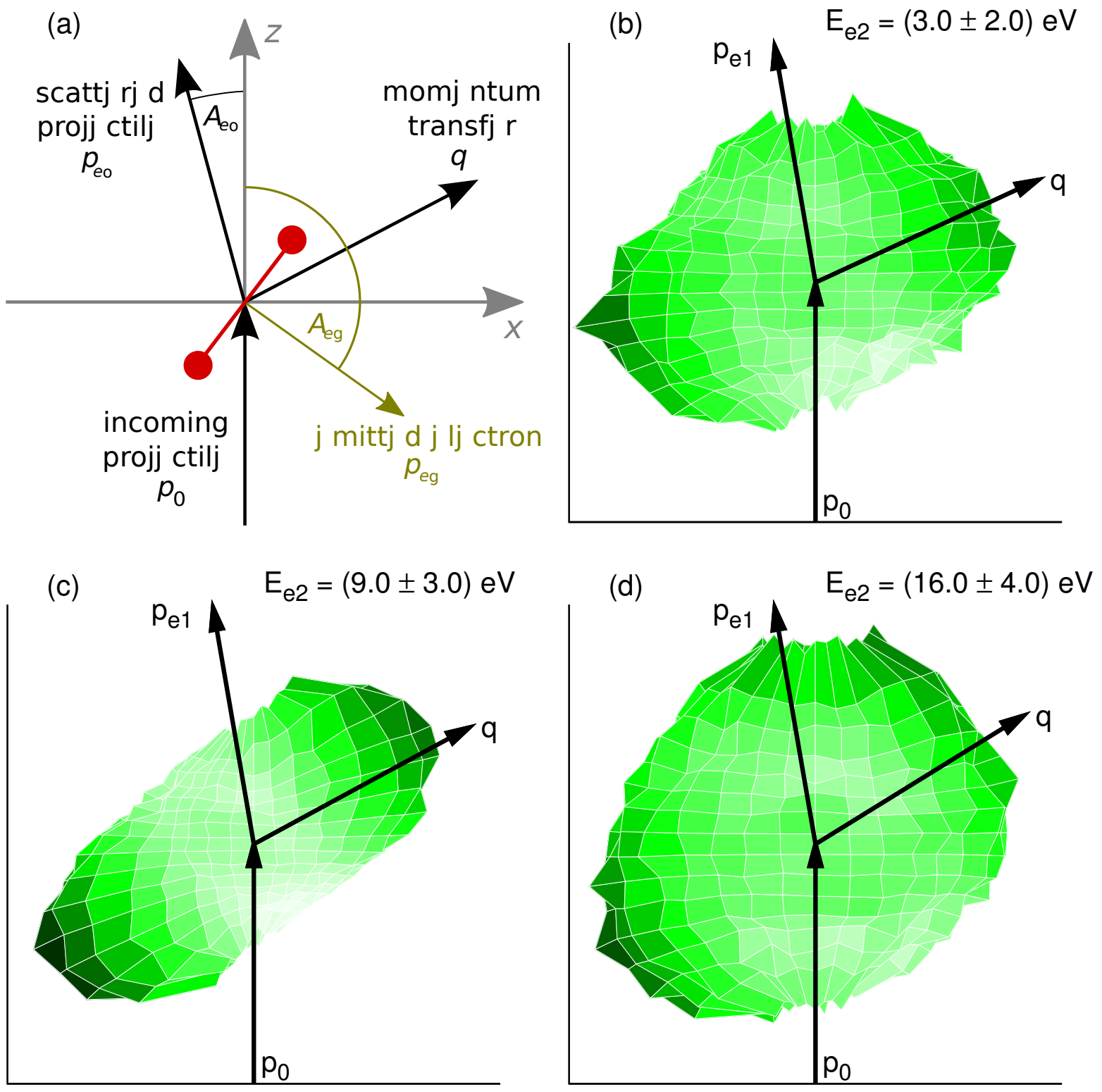

Figure 3. (colour online) (a) Geometry of the ionizing collision in the scattering plane spanned by the incoming and scattered projectile momentum vectors. An exemplary momentum of the emitted electron is sketched. (b)-(d) Dependence of the ionization cross section on the emission direction of the protonic fragment. Integrated over the whole detected solid angle for the two electrons and proton kinetic energy releases between .13 and $1 \mathrm{eV}$ while the emitted electron energy amounts (b) (3 \pm 2$) \mathrm{eV}$, (c) $(9 \pm 3) \mathrm{eV}$ and $(\mathrm{d})(16 \pm 4) \mathrm{eV}$. 

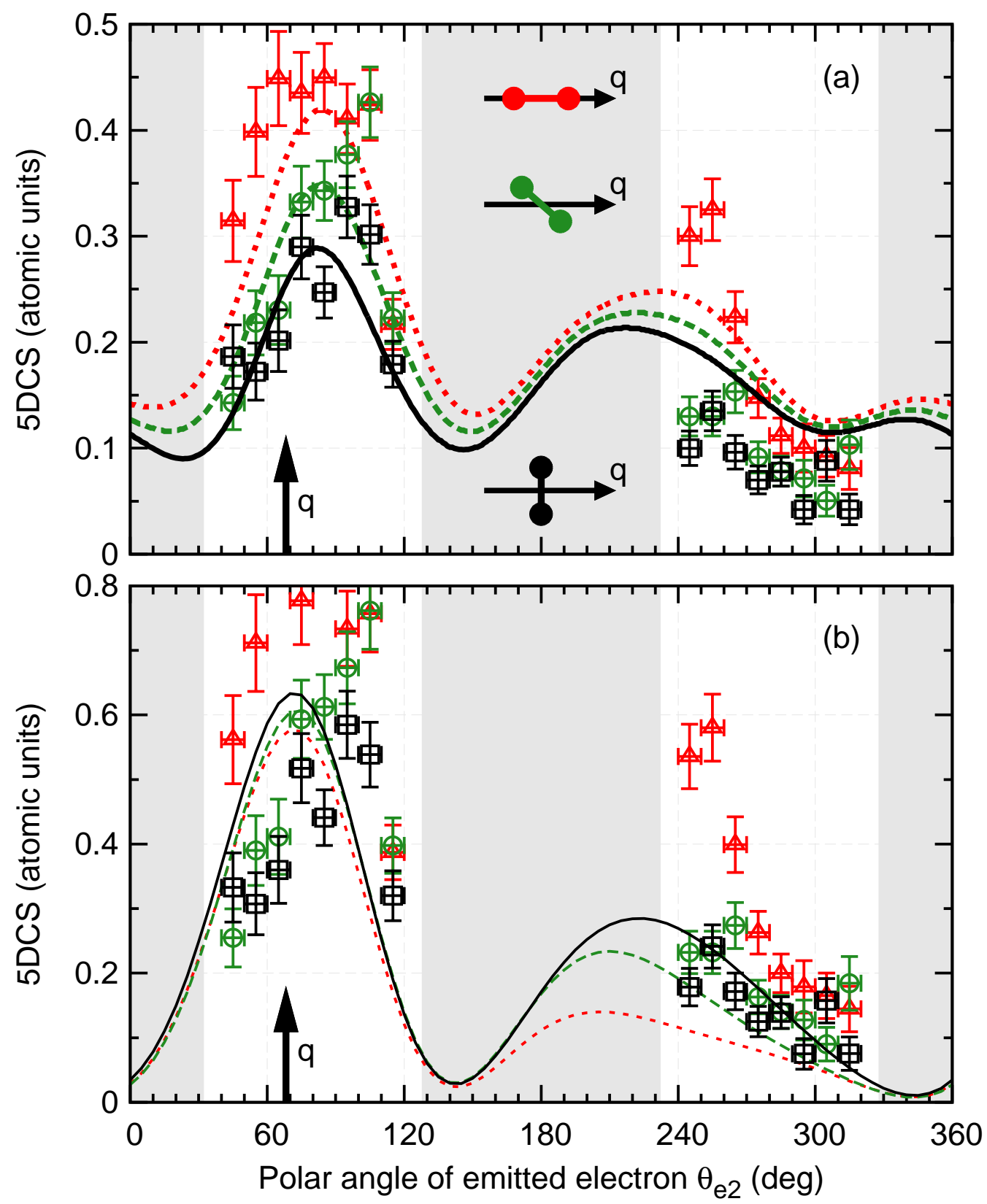

Figure 4. Five-fold differential cross sections (5DCS) as a function of the emitted electron's emission angle in the scattering plane. This electron's energy is $(3.5 \pm 2.0) \mathrm{eV}$ and the projectile scattering angle is $(16 \pm 4)^{\circ}$. Points represent experimental results, lines model calculations, which is either (a) molecular 3-body distorted wave or (b) the three-Coulomb wavefunction approach for a helium target multiplied with the interference factor given by (Stia et al. 2003). For all data shown the molecule is aligned in the scattering plane, at angles of $0^{\circ}$ (triangles/dotted line), $45^{\circ}$ (circles/dashed line) or $90^{\circ}$ (squares/solid line) relative to the momentum transfer $\vec{q}$. Shaded areas represent angular ranges where the electron cannot be detected due to the beam dump in the center of the detector. 
transfer. For all molecular alignments, the spectra resemble classical $(e, 2 e)$ spectra for the ionization of an atomic s-state (compare e.g. (Dürr et al. 2006)). Generally, a reasonable agreement between $\mathrm{M} 3 \mathrm{DW}$ calculation and experiment is found, especially in the binary region below $180^{\circ}$ where the distinct experimental alignment-dependence is well reproduced. At essentially all electron emission angles, parallel alignment of the molecule with respect to momentum transfer employs the highest cross-sections, while the perpendicular case features the lowest. An explicit disagreement between M3DW and experiment is found around $250^{\circ}$, where parallel alignment was found to show even higher rates. The origin of this is still not understood. At much lower energies, the timedependent close-coupling (TDCC) method has recently predicted such strongly varying 5DCS for different molecular alignments. On the other hand, it has to be expected that in the dipole limit at very high electron energies, the ionization cross sections for GSD will become independent of the molecular alignment, as reported in photoionization experiments (Hikosaka \& Eland 2003, Lafosse et al. 2003). In the current case we seem to be in an intermediate regime where the location of the nuclei starts to play a role in the collision. In addition, we have to note the enhanced experimental cross-section on the left side of the binary peak for parallel alignment. This makes said peak look like having its maximum in the direction of $\vec{q}$. Normally, at the kinematics studied here, one would expect the centre of the binary lobe a few degrees above the momentum transfer, due to post-collision interaction. We observe this in the calculation and also in the experimental data for larger angles between $\vec{q}$ and the molecular axis. The discrepancy in the parallel case could be due to a small contribution of autoionization that might not be completely excluded at this kinematics.

We have also investigated the experimental results in terms of the two-centre picture developed by (Stia et al. 2003) that predicts interference effects. Hereby, 5DCS are obtained by multiplying triply differential cross sections (3DCS) for an atomic target with the interference factor

$$
I=2\left[1+\cos \left(\left(\vec{q}-\vec{p}_{e 2}\right) \cdot \vec{R}\right)\right]
$$

depending on the molecular alignment $\vec{R}$. To demonstrate the effect of $I$ we have currently employed 3DCS for the two-electron system helium calculated using the threeCoulomb (3C) wavefunction approach which was found to be in reasonable agreement to experimental data (Dürr et al. 2006). The resulting 5DCS are displayed in figure 4 (b). Once again, the measured cross sections have been normalized to the calculations at the theoretical maximum of the $45^{\circ}$ case. Apparently, this model disagrees significantly with the experimental results. First, the cross sections of the distinct molecular alignments are reversed in order, i.e. the interference factor predicts highest probabilities when the molecule is perpendicular to momentum transfer. This discrepancy is most obvious in the recoil lobe. Additionally, the model exhibits much smaller overall alignmentdependence in the binary region than observed. From this we conclude that interference, at least in terms of Stia's model, is not able to explain the observed cross sections for ground-state ionization of $\mathrm{H}_{2}$ at $200 \mathrm{eV}$ impact energy. By having the additional 
information of alignment we can see that Stia's model is inadequate while it was found to reproduce the binary-to-recoil ratio in experiments with randomly orientated molecules at a comparable impact energy (Milne-Brownlie et al. 2006). However, interference effects might certainly be present in the ionization of $\mathrm{H}_{2}$ in a more subtle way and should be accounted for implicitly by the M3DW calculation.

To summarize, five-fold differential cross sections of electron impact ionization of molecular hydrogen have been successfully measured. Two dissociation channels leading to low-energetic protons could be identified: Ground-state dissociation and autoionization. For GSD, experimental data was well matched by M3DW calculation, although unexplained discrepancies remain. On the other hand, atomic crosssections multiplied with a alignment-dependent interference factor failed to reproduce experimental 5DCS.

\section{Acknowledgements}

This work was partly supported by the USA National Science Foundation under Grant. No. PHY-0757749. The author OA-H would like to acknowledge the support of the Saudi Ministry of Higher Education's King Abdullah Bin Abdul-Aziz Scholarship. XR is grateful for support from DFG project No. RE 2966/1-1.

\section{References}

Al-Hagan O, Kaiser C, Madison D H \& Murray A J 2008 Nat. Phys. 5, 59.

Bellm S, Lower J, Weigold E \& Mueller D W 2010 Phys. Rev. Lett. 104, 023202.

Cherid M, Lahmam-Bennani A, Dugett A, Zurales R W, Lucchese R R, Dal Cappello M C \& Dal Cappello C 1989 J. Phys. B: At. Mol. Opt. Phys. 22, 3483-3499.

Colgan J, Al-Hagan O, Madison D H, Murray A J \& Pindzola M S 2009 J. Phys. B: At. Mol. Opt. Phys. 42, 171001.

Colgan J, Pindzola M S, Robicheaux F, Kaiser C, Murray A J \& Madison D H 2008 Phys. Rev. Lett. 101, 233201.

Dorn A, Dürr M, Najjari B, Haag N, Dimopoulou C, Nandi D \& Ullrich J 2007 J. Electron Spectrosc. Relat. Phenom. 161, 2-5.

Dunn G H \& Kieffer L J 1963 Phys. Rev. 132, 2109-2117.

Dürr M, Dimopoulou C, Dorn A, Najjari B, Bray I, Fursa D V, Chen Z, Madison D H, Bartschat K \& Ullrich J 2006 J. Phys. B: At. Mol. Opt. Phys. 39, 4097-4111.

Dürr M, Dorn A, Ullrich J, Cao S P, Czasch A, Kheifets A S, Götz J R \& Briggs J S 2007 Physical Review Letters 98(19), 193201.

Edwards A K, Wood R M, Davis J L \& Ezell R L 1990 Phys. Rev. A 42, 1367-1375.

Edwards A K \& Zheng Q 2001 J. Phys. B: At. Mol. Opt. Phys. 34, 1539-1548.

Gao J, Madison D H, Peacher J L, Murray A J \& Hussey M J H 2006 J. Chem. Phys. 124, 194306.

Guberman S L 1983 J. Chem. Phys. 78, 1404-1413.

Hikosaka Y \& Eland J H D 2003 J. Electron Spectrosc. Relat. Phenom. 133, 77-86.

Jagutzki O, Cerezo A, Czasch A, Dörner R, Hattass M, Huang M, Mergel V, Spillmann U, UllmannPfleger K, Weber T, Schmidt-Böcking H \& Smith G D W 2002 IEEE T. Nucl. Sci. 49, 2477-2483.

Lafosse A, Lebech M, Brenot J C, Guyon P M, Spielberger L, Jagutzki O, Houver J C \& Dowek D 2003 J. Phys. B: At. Mol. Opt. Phys. 36, 4683-4702. 
Laurent G, Fernández J, Legendre S, Tarisien M, Adoui L, Cassimi A, Fléchard X, Frémont F, Gervais B, Giglio E, Grandin J P \& Martín F 2006 Phys. Rev. Lett. 96, 173201.

Milne-Brownlie D S, Foster M, Gao J, Lohmann B \& Madison D H 2006 Phys. Rev. Lett. 96, 233201. Murray A J 2005 J. Phys. B: At. Mol. Opt. Phys. 38, 1999-2013.

Pedersen H B, Altevogt S, Jordon-Thaden B, Heber O, Lammich L, Rappaport M L, Schwalm D, Ullrich J, Zajfmann D, Treusch R, Guerassimova N, Martins M \& Wolf A 2009 Phys. Rev. A 80, 012707.

Sharp T E 1970 At. Data Nucl. Data Tables 2, 119-169.

Staicu Cassagrande E M, Naja A, Mezdari F, Lahmam-Bennani A, Bolognesi P, Joulakian B, Chuluunbaatar O, Al-Hagan O, Madison D H, Fursa D V \& Bray I 2008 J. Phys. B: At. Mol. Opt. Phys. 41, 052701.

Stia C R, Fójon O A, Weck P F, Hanssen J \& Rivarola R D 2003 J. Phys. B: At. Mol. Opt. Phys. 36, L257-264.

Takahashi M, Watanabe N, Khajuria Y, Nakayama K, Udagawa Y \& Eland J H D 2004 J. Electron Spectrosc. Relat. Phenom. 141, 83-93.

Takahashi M, Watanabe N, Khajuria Y, Udagawa Y \& Eland J H D 2005 Phys. Rev. Lett. 94, 213202. Van Brunt R J \& Kieffer L J 1970 Phys. Rev. A 2, 1293-1304.

Van Zyl B \& Stephene T M 1994 Phys. Rev. A 50, 3164.

Weigold E, Hood S T, McCarthy I E \& Teubner P J O 1973 Phys. Lett. A 44, 531-532.

Wood R M, Zheng Q, Edwards A K \& Mangan M A 1997 Rev. Sci. Instrum. 68, 1382-1386.

Zare R N 1967 J. Chem. Phys. 47, 204-215. 